\title{
Interval Sieve Algorithm \\ Creating a Countable Set of Real Numbers from a Closed Interval
}

(C) 2019 by Ron Ragusa All rights reserved

\section{Abstract}

The Interval Sieve Algorithm is a method for generating a list of real numbers on any closed interval of real numbers $\left[r_{i}, r_{j}\right]$ where $r_{i}<r_{j}$. Georg Cantor in his 1891 paper demonstrated a constructive proof that the real numbers are uncountable. Cantor developed a method for showing how a particular objective cannot be accomplished; in this case, establishing a one to one correspondence between the natural numbers and the real numbers, using his diagonal argument.

What Cantor didn't show is that there are no ways of demonstrating a one to one correspondence between the natural numbers and the real numbers. This is important because even if one can demonstrate one or more ways that something cannot be done, it is only necessary to develop one way that shows how it can be done to invalidate the ways that show it cannot.

The interval sieve algorithm partitions a closed interval of real numbers $\left[r_{i}, r_{j}\right]$ where $r_{i}<r_{j}$ to create a complete list, $\mathrm{L}$, of numbers in the interval. We will prove that the list $\mathrm{L}$ is complete, and derive the bijective function $f: \mathbb{N} \rightarrow\left[\mathrm{r}_{1}, \mathrm{r}_{2}\right]$.

\section{Definitions}

1. The lower bound of an interval is the leftmost member of the interval. In the interval $\left[r_{1}, r_{2}\right], r_{1}$ is the lower bound of the interval.

2. The upper bound of an interval is the rightmost member of the interval. In the interval $\left[\mathrm{r}_{1}, \mathrm{r}_{2}\right], \mathrm{r}_{2}$ is the upper bound of the interval.

3. Given the set, $S=\{1,2,3\}$. We define a closed interval of the set as $I_{S}=[1,3]$ where both upper and lower bounds are included in the interval.

4. Given the set, $S=\{1,2,3\}$. We define an open interval of the set as $I_{S}=(1,3)$ where the upper and lower bounds are excluded from the interval.

5. Given the set, $S=\{1,2,3\}$. We define a lower open interval of the set as $I_{S}=(1,3]$ where the lower bound is excluded from the interval and the upper bound is included in the interval.

6. Given the set, $S=\{1,2,3\}$. We define an upper open interval of the set as $I_{S}=[1,2)$ where the lower bound is included in the interval and the upper bound is excluded from the interval.

7. A conjoined interval pair is a pair of intervals where the upper bound of one and the lower bound of the other are the same member. $\left[\mathrm{r}_{\mathrm{i}},\left[\mathrm{r}_{\mathrm{k}},\right] \mathrm{r}_{\mathrm{j}}\right]$ is an example of a conjoined interval pair where $r_{k}$ is both the upper bound of $\left[r_{i}, r_{k}\right]$, the lower bound of $\left[r_{k}, r_{j}\right]$ and $r_{i}<r_{k}<r_{j}$. 
8. A relative bound is the number that is common to both intervals in a conjoined interval pair. In the conjoined interval pair $\left[\mathrm{r}_{1},\left[\mathrm{r}_{3},\right] \mathrm{r}_{2}\right], \mathrm{r}_{3}$ is the relative bound in both intervals $\left[\mathrm{r}_{1}, \mathrm{r}_{3}\right]$ and $\left[\mathrm{r}_{3}, \mathrm{r}_{2}\right]$.

9. An interval of a set may be partitioned by creating a conjoined interval pair per definition 7 and then splitting the conjoined interval pair into sub-intervals with the relative bound being the upper bound of one sub-interval and the lower bound of the other sub-interval.

Example:

$\mathrm{S}=\{1,2,3\}$

$\mathrm{I}_{\mathrm{S}}=[1,3] \quad\left(\mathrm{I}_{\mathrm{S}} \longrightarrow\right.$ the interval I on set $\left.\mathrm{S}\right)$

Partition $\mathrm{I}_{\mathrm{S}}$ as follows -

$\mathrm{I}_{\mathrm{S}}=[1,3]$

$=[1,[2], 3]$

$=[1,2],[2,3]$

10. When no sub-intervals can be further subdivided then the interval is called fully partitioned.

11. The immediate predecessor of a number $\lambda$ is a number $\beta$ such that there exists no number $\delta$ where $\beta<\delta<\lambda$.

12. The immediate successor of a number $\lambda$ is a number $\beta$ such that there exists no number $\delta$ where $\lambda<\delta<\beta$.

13. For any 2 real numbers $\lambda$ and $\beta$ in $\left[\mathrm{r}_{1}, \mathrm{r}_{2}\right]$, we can always find another real number, $\delta$, such that if $\lambda>\beta$ then $\beta<\delta<\lambda$ and if $\lambda<\beta$ then $\lambda<\delta<\beta$. Therefore from definitions 11 and 12 we know that there are no immediate predecessors or successors of any of the elements of $\left[\mathrm{r}_{1}, \mathrm{r}_{2}\right]$; that is, $\left[\mathrm{r}_{1}, \mathrm{r}_{2}\right]$ is a continuum.

\section{The Interval Sieve Algorithm}

\section{Interval Sieve Algorithm}

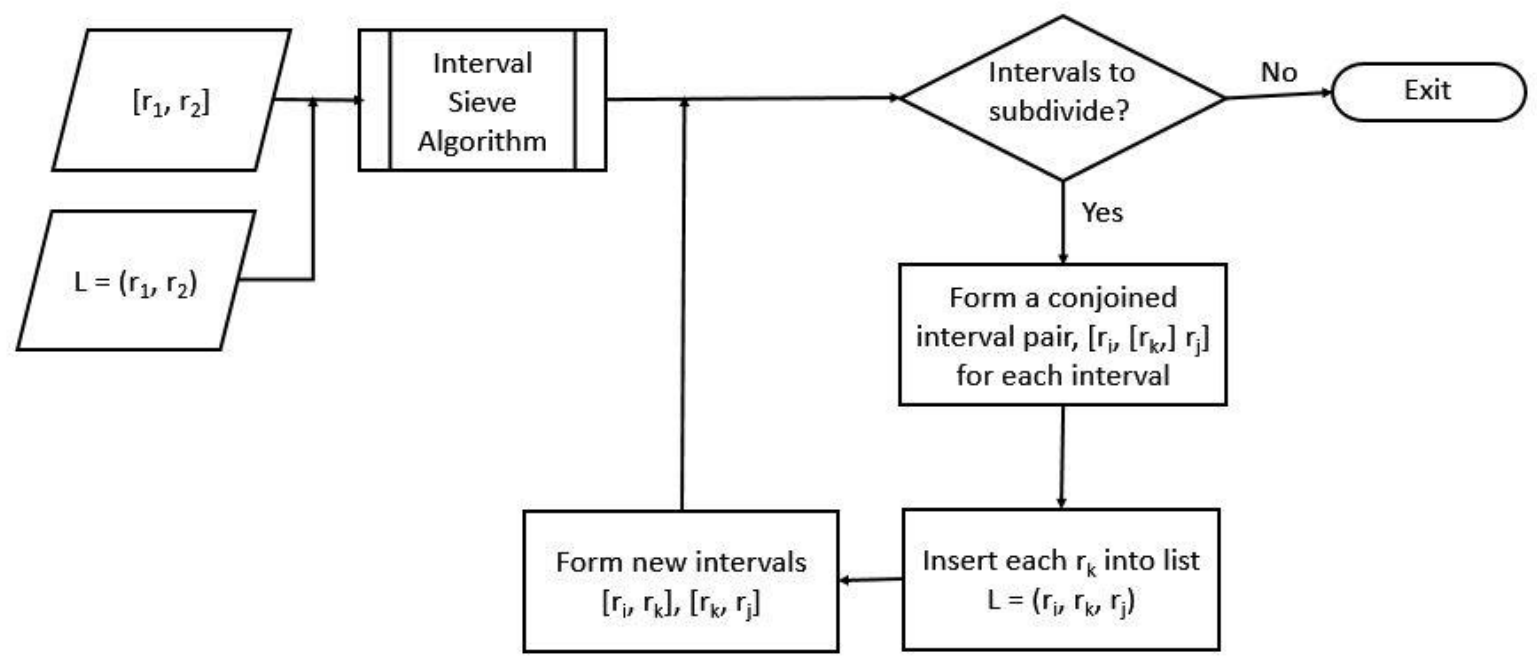


Procedure:

0 . We begin the procedure given the interval $\left[r_{1}, r_{2}\right]$ where $r_{1}<r_{2}$ and $r_{1}, r_{2}$ are real numbers and the list $\mathrm{L}=\left(\mathrm{r}_{1}, \mathrm{r}_{2}\right)$.

1. If there are intervals that can be sub-divided next step else stop..

2. Sub-divide each interval $\left[r_{i}, r_{j}\right]$ by selecting a number $r_{k}$ such that $r_{i}<r_{k}<r_{j}$ to get a conjoined interval pair $\left[\mathrm{r}_{\mathrm{i}},\left[\mathrm{r}_{\mathrm{k}},\right] \mathrm{r}_{\mathrm{i}}\right]$

3. Insert the relative bound number, $r_{k}$, into the list $L$ to get $L=\left(r_{i}, r_{k}, r_{i}\right)$.

4. Form new sub-intervals $\left[\mathrm{r}_{\mathrm{i}}, \mathrm{r}_{\mathrm{k}}\right],\left[\mathrm{r}_{\mathrm{k}}, \mathrm{r}_{\mathrm{j}}\right]$.

5. Return to step 1.

\section{Partitioning Intervals Using the Algorithm}

We will use the partitioning of a set interval according to the algorithm as a method for creating a list of the elements of the interval. Partitioning allows us to construct a list whereby all the elements of the interval will be included in the list.

Below are two examples of partitioning set intervals.

Example 1 -

Let:

$S=\{1,2,3,4,5\}$

$\mathrm{I}_{\mathrm{S}}=[1,5]$

First we note that in defining the interval $[1,5]$ we have specified the first two elements of the list L. That is $L=(1,5)$. To continue populating $L$, partition $[1,5]$, adding each relative bound to $L$ as it is created, and continue the process until no intervals are left that can be partitioned.

$$
\begin{aligned}
\mathrm{I}_{\mathrm{S}} & =[1,5] & \mathrm{L}=(1,5) \\
& =[1,[3], 5] & \mathrm{L}=(1,3,5) \\
& =[1,3],[3,5] & \\
& =[1,[2], 3],[3,[4], 5] & \mathrm{L}=(1,2,3,4,5) \\
& =[1,2],[2,3],[3,4],[4,5] &
\end{aligned}
$$

Since no interval in $\mathrm{I}_{\mathrm{S}}$ can be further subdivided the interval is fully partitioned and $\mathrm{L}$ is complete.

Example 2 -

Let:

$S=\{1,2,3, \ldots \omega\} \quad$ where $\omega$ is the first infinite ordinal number. 
$\mathrm{I}_{\mathrm{S}}=[1, \omega)$

Partition the interval $[1, \omega)$ to create a list $\mathrm{L}$ of natural numbers.

$$
\begin{aligned}
\mathrm{I}_{\mathrm{S}} & =[1, \omega) & \mathrm{L}=(1) \\
& =[1,[2], \omega) & \mathrm{L}=(1,2) \\
& =[1,2],[2, \omega) & \\
& =[1,2],[2,[3], \omega) & \mathrm{L}=(1,2,3) \\
& =[1,2],[2,3],[3, \omega) &
\end{aligned}
$$

Taking the procedure to its limit will create a list of all the natural numbers, $\mathrm{L}=(1,2,3, \ldots)$. Since $\mathrm{I}_{\mathrm{S}}$ is defined as an upper open interval, $\omega$ is not included in $\mathrm{L}$.

\section{Creating $L$ over $\left[\mathbf{r}_{1}, \mathbf{r}_{2}\right]$ where $\mathbf{r}_{1}, \mathbf{r}_{2}$ are Real Numbers}

Let:

$S=\{\mathbb{R}\}$

$\mathrm{I}_{\mathrm{S}}=\left[\mathrm{r}_{1}, \mathrm{r}_{2}\right]$ where $\mathrm{r}_{1}<\mathrm{r}_{2}$

Partition $I_{S}$ to create the list $L$ of real numbers between $r_{1}$ and $r_{2}$.

$$
\begin{array}{rlrl}
\mathrm{I}_{\mathrm{S}} & =\left[\mathrm{r}_{1}, \mathrm{r}_{2}\right] & \mathrm{L}=\left(\mathrm{r}_{1}, \mathrm{r}_{2}\right) & \mathrm{L}=\left(\mathrm{r}_{1}, \mathrm{r}_{3}, \mathrm{r}_{2}\right) \\
& =\left[\mathrm{r}_{1},\left[\mathrm{r}_{3}\right], \mathrm{r}_{2}\right] & \\
& =\left[\mathrm{r}_{1}, \mathrm{r}_{3}\right],\left[\mathrm{r}_{3}, \mathrm{r}_{2}\right] & \mathrm{L}=\left(\mathrm{r}_{1}, \mathrm{r}_{4}, \mathrm{r}_{3}, \mathrm{r}_{5}, \mathrm{r}_{2}\right) \\
& =\left[\mathrm{r}_{1},\left[\mathrm{r}_{4}\right], \mathrm{r}_{3}\right],\left[\mathrm{r}_{3},\left[\mathrm{r}_{5}\right], \mathrm{r}_{2}\right] & \\
& =\left[\mathrm{r}_{1}, \mathrm{r}_{4}\right],\left[\mathrm{r}_{4}, \mathrm{r}_{3}\right],\left[\mathrm{r}_{3}, \mathrm{r}_{5}\right],\left[\mathrm{r}_{5}, \mathrm{r}_{2}\right] & \mathrm{L}=\left(\mathrm{r}_{1}, \mathrm{r}_{6}, \mathrm{r}_{4}, \mathrm{r}_{7}, \mathrm{r}_{3}, \mathrm{r}_{8}, \mathrm{r}_{5}, \mathrm{r}_{9}, \mathrm{r}_{2}\right) \\
& =\left[\mathrm{r}_{1},\left[\mathrm{r}_{6}\right], \mathrm{r}_{4}\right],\left[\mathrm{r}_{4},\left[\mathrm{r}_{7}\right], \mathrm{r}_{3}\right],\left[\mathrm{r}_{3},\left[\mathrm{r}_{8}\right], \mathrm{r}_{5}\right],\left[\mathrm{r}_{5},\left[\mathrm{r}_{9}\right], \mathrm{r}_{2}\right] & \\
& =\left[\mathrm{r}_{1}, \mathrm{r}_{6}\right],\left[\mathrm{r}_{6}, \mathrm{r}_{4}\right],\left[\mathrm{r}_{4}, \mathrm{r}_{7}\right],\left[\mathrm{r}_{7}, \mathrm{r}_{3}\right],\left[\mathrm{r}_{3}, \mathrm{r}_{8}\right],\left[\mathrm{r}_{8}, \mathrm{r}_{5}\right],\left[\mathrm{r}_{5}, \mathrm{r}_{9}\right],\left[\mathrm{r}_{9}, \mathrm{r}_{2}\right] &
\end{array}
$$

At the limit of the process $L$ will appear as follows: $L=\left(r_{1}, \ldots r_{6}, \ldots r_{4}, \ldots r_{7}, \ldots r_{3}, \ldots r_{8}, \ldots r_{5}, \ldots r_{9}, \ldots r_{2}\right)$.

By definition 13 there are no immediate predecessors or successors in $\left[\mathrm{r}_{1}, \mathrm{r}_{2}\right]$. It follows that the partitioning of sub-intervals of $\left[r_{1}, r_{2}\right]$ can go on indefinitely. Also, except for $r_{1}$ and $r_{2}$, every number in the original interval must, at some point during the process, become a relative bound and only then added to L. And because no number will be a relative bound more than once, there will be no duplicates in $\mathrm{L}$.

As can be seen in the examples above, each relative bound becomes the lower bound of one subinterval and the upper bound of another sub-interval. This means that every number in the each 
sub-interval will be approached from left below and right its value and the interval lengths will become infinitesimally small.

Example 3, using numbers:

Let:

$\mathrm{S}=\{\mathbb{R}\}$

$\mathrm{I}_{\mathrm{S}}=[1,4]$

Partition $\mathrm{I}_{\mathrm{S}}$ to create the list $\mathrm{L}$ of real numbers between 1 and 4.

$$
\begin{array}{rlrl}
\mathrm{I}_{\mathrm{S}} & =[1,4] & \mathrm{L}=(1,4) \\
& =[1,[\pi], 4] & \mathrm{L}=(1, \pi, 4) \\
& =[1, \pi],[\pi, 4] & \\
& =[1,[e], \pi],[\pi,[3.2], 4] & \mathrm{L}=(1, e, \pi, 3.2,4) \\
& =[1, e],[e, \pi],[\pi, 3.2],[3.2,4] & \\
& =[1,[\sqrt{ } 2], e],[e,[3], \pi],[\pi,[3.15], 3.2],[3.2,[3.3], 4] & \mathrm{L}=(1, \sqrt{ } 2, e, 3, \pi, 3.15,3.2,3.3,4) \\
& =[1, \sqrt{ } 2],[\sqrt{ } 2, e],[e, 3],[3, \pi],[\pi, 3.15],[3.15,3.2],[3.2,3.3],[3.3,4]
\end{array}
$$

At the limit of the process $L=(1, \ldots \sqrt{ } 2, \ldots e, \ldots 3, \ldots \pi, \ldots 3.15, \ldots 3.2, \ldots 3.3, \ldots 4)$.

\section{Proving the List $\mathrm{L}$ is Complete}

The question remains as to whether or not the list $\mathrm{L}$ will contain all real numbers in $\left[\mathrm{r}_{1}, \mathrm{r}_{2}\right]$. We will prove that: All the real numbers in $\left[\mathrm{r}_{1}, \mathrm{r}_{2}\right]$ are contained in the list $\mathrm{L}$.

Proof by Construction/Contradiction: Create a number $\mathrm{X}$ such that $\mathrm{r}_{1}<\mathrm{X}<\mathrm{r}_{2}$ and assume that $\mathrm{X} \notin \mathrm{L}$. Demonstrate that ' $\mathrm{r}_{1}<\mathrm{X}<\mathrm{r}_{2}$ and that $\mathrm{X} \notin \mathrm{L}$ ' leads to a contradiction.

To create X we employ Cantor's diagonal method:

\begin{tabular}{l|ccccccccc} 
Row & \multicolumn{10}{|c}{$\left[\mathrm{r}_{1}, \mathrm{r}_{2}\right]$} & & & & \\
\hline 1 & &. $\mathrm{~d}_{1}$ & $\mathrm{~d}_{2}$ & $\mathrm{~d}_{3}$ & $\mathrm{~d}_{4}$ & $\mathrm{~d}_{5}$ & $\mathrm{~d}_{6}$ & $\mathrm{~d}_{7}$ & $\ldots$ \\
2 & &. $\mathrm{~d}_{1}$ & $\mathrm{~d}_{2}$ & $\mathrm{~d}_{3}$ & $\mathrm{~d}_{4}$ & $\mathrm{~d}_{5}$ & $\mathrm{~d}_{6}$ & $\mathrm{~d}_{7}$ & $\ldots$ \\
3 & & $\mathrm{~d}_{1}$ & $\mathrm{~d}_{2}$ & $\mathrm{~d}_{3}$ & $\mathrm{~d}_{4}$ & $\mathrm{~d}_{5}$ & $\mathrm{~d}_{6}$ & $\mathrm{~d}_{7}$ & $\ldots$ \\
4 & & $\mathrm{~d}_{1}$ & $\mathrm{~d}_{2}$ & $\mathrm{~d}_{3}$ & $\mathrm{~d}_{4}$ & $\mathrm{~d}_{5}$ & $\mathrm{~d}_{6}$ & $\mathrm{~d}_{7}$ & $\ldots$ \\
5 & &. $\mathrm{~d}_{1}$ & $\mathrm{~d}_{2}$ & $\mathrm{~d}_{3}$ & $\mathrm{~d}_{4}$ & $\mathrm{~d}_{5}$ & $\mathrm{~d}_{6}$ & $\mathrm{~d}_{7}$ & $\ldots$ \\
6 & &. $\mathrm{~d}_{1}$ & $\mathrm{~d}_{2}$ & $\mathrm{~d}_{3}$ & $\mathrm{~d}_{4}$ & $\mathrm{~d}_{5}$ & $\mathrm{~d}_{6}$ & $\mathrm{~d}_{7}$ & $\ldots$ \\
7 & & $\mathrm{~d}_{1}$ & $\mathrm{~d}_{2}$ & $\mathrm{~d}_{3}$ & $\mathrm{~d}_{4}$ & $\mathrm{~d}_{5}$ & $\mathrm{~d}_{6}$ & $\mathrm{~d}_{7}$ & $\ldots$ \\
$\ldots$ & & & & $\ldots$ & & & & & \\
& $\mathrm{X}=$ & $\mathrm{b}_{1}$ & $\mathrm{~b}_{2}$ & $\mathrm{~b}_{3}$ & $\mathrm{~b}_{4}$ & $\mathrm{~b}_{5}$ & $\mathrm{~b}_{6}$ & $\mathrm{~b}_{7}$ & $\ldots$
\end{tabular}


Each $b_{i}$ of $X$ differs from each number in the list at $d_{i}$. Using the diagonal method we have created a number $\mathrm{X}$ such that $\mathrm{r}_{1}<\mathrm{X}<\mathrm{r}_{2}$ and $\mathrm{X} \notin \mathrm{L}$ (Cantor's proof).

1. Since $X$ is an element of $\left[r_{1}, r_{2}\right]$ we can rewrite $\left[r_{1}, r_{2}\right]$ as $\left[r_{1}, \ldots X, \ldots r_{2}\right]$.

2. We can now select $X$ as the first cut point in the partitioning of $\left[r_{1}, r_{2}\right]$ and form the relative bound of the conjoined interval pair $\left[\mathrm{r}_{1},[\mathrm{X}], \mathrm{r}_{2}\right]$.

3. Once $\mathrm{X}$ is designated a relative bound it will be inserted into $\mathrm{L}$ per the algorithm and we'll have $\mathrm{L}=\left(\mathrm{r}_{1}, \mathrm{X}, \mathrm{r}_{2}\right)$.

4. Having shown that $\mathrm{X} \in \mathrm{L}$, we can say that the original assertion, $\mathrm{X} \notin \mathrm{L}$, leads to a contradiction and must be false.

5. Since the list of $\left[r_{1}, r_{2}\right]$ created using the diagonal method was lacking $X$, and we have shown that by partitioning $\left[r_{1}, r_{2}\right]$ using the interval sieve includes $X$ as a member of the list of $\left[r_{1}, r_{2}\right]$ we can assert that, at the limit, $\mathrm{L}$ will be complete and this ends the proof.

\section{Derivation of $f: \mathbb{N} \rightarrow\left[\mathrm{r}_{1}, \mathbf{r}_{2}\right]$}

We will now demonstrate that there exists a bijective function from $\mathbb{N}$ to $\left[\mathrm{r}_{1}, \mathrm{r}_{2}\right], f: \mathbb{N} \rightarrow\left[\mathrm{r}_{1}, \mathrm{r}_{2}\right]$.

We have used the Interval Sieve Algorithm to create: $L=\left(r_{1}, \ldots r_{6}, \ldots r_{4}, \ldots r_{7}, \ldots r_{3}, \ldots r_{8}, \ldots r_{5}, \ldots r_{9}, \ldots r_{2}\right)$

and have proved $\mathrm{L}$ is complete. It is readily apparent that for every $\mathrm{r}$ in the list there is an associated natural number subscript. Since $\mathrm{L}$ is complete, containing all numbers in $\left[\mathrm{r}_{1}, \mathrm{r}_{2}\right]$ and each number in $\mathrm{L}$ is associated with a single unique natural number we can assert that $f: \mathbb{N} \rightarrow\left[\mathrm{r}_{1}, \mathrm{r}_{2}\right]$ exists.

The existence of $f: \mathbb{N} \rightarrow\left[\mathrm{r}_{1}, \mathrm{r}_{2}\right]$ confirms a one to one correspondence between the natural numbers and any closed interval of real numbers.

\section{Final Thoughts}

The fact that we have shown the existence of $f: \mathbb{N} \rightarrow\left[\mathrm{r}_{1}, \mathrm{r}_{2}\right]$ implies that Cantor's continuum hypothesis is true for closed intervals of real numbers. Interestingly, it's not that there are no infinite sets with cardinality between $\boldsymbol{\aleph}_{0}$ and $\boldsymbol{\aleph}_{1}$, rather $\mathbb{N}$ and $\left[\mathrm{r}_{1}, \mathrm{r}_{2}\right]$ turn out to be the same size. 